\title{
Comparison of Adjuvant Chemotherapy and Chemoradiation Therapy Efficacy for Pancreatic Cancer After RO Resection: A Propensity Score- Matched Analysis
}

\section{Min su You}

Seoul National University Hospital

Jikon Ryu ( $\nabla$ jkryu@snu.ac.kr)

Seoul National University College of Medicine https://orcid.org/0000-0001-8798-0491

Young Hoon Choi

Seoul National University Hospital

\section{Bang-sup Shin}

Seoul National University Hospital

\section{Woo Hyun Paik}

Seoul National University Hospital

\section{Sang Hyub Lee}

Seoul National University Hospital

\section{Yong-Tae Kim}

Seoul National University Hospital

\section{Research article}

Keywords: pancreatic cancer, adjuvant chemotherapy, adjuvant chemoradiotherapy, treatment efficacy, adverse effects

Posted Date: August 2nd, 2019

DOI: https://doi.org/10.21203/rs.2.12295/v1

License: (c) (1) This work is licensed under a Creative Commons Attribution 4.0 International License. Read Full License 


\section{Abstract}

Background Adjuvant therapy is a standard treatment for resected pancreatic cancer, and a variety of regimens are now used in clinical practice. We aimed to compare the efficacy of adjuvant chemotherapy and chemoradiation therapy in R0 resected pancreatic cancer. Methods Patients who underwent complete microscopic resection and subsequent adjuvant treatment for pancreatic ductal adenocarcinoma ( $\mathrm{N}=277$ ) at Seoul National University Hospital from September 2005 to December 2017 were retrospectively included. Patients were divided into two groups; systemic chemotherapy (SCT) and combined chemotherapy plus chemoradiation therapy (SCT-CRT). The primary outcomes were differences in overall survival (OS) between the two groups. Survival differences were compared using the log-rank test. Patients receiving either SCT or SCT-CRT were matched 1:1 on the propensity scores. Results Patients received SCT $(n=78)$ and SCT-CRT $(n=199)$. There was no significant difference in completion rate of planned regimens between the two groups ( $73.1 \%$ and $76.9 \%$, respectively; $P=0.534)$. In the propensity score-matched cohort, there was no significant difference in the median OS between the SCT and SCT-CRT groups (35.1 [95\% Cl, 26.9-82.1] months and 34.8 [95\% Cl, 26.2-69.2] months, respectively; $\mathrm{P}=0.993)$. The median RFS was $15.4(95 \% \mathrm{Cl}, 12.1-31.1)$ months in the SCT group and 17.0 $(95 \% \mathrm{Cl}, 13.2-37.3)$ months in SCT-CRT group ( $P=0.789)$. There were no significant differences in the incidence of loco-regional and distant recurrence between the two groups $(P=0.833$ and $P=0.491$, respectively). Adverse events greater than or equal to moderate grade occurred more frequently in the SCT-CRT group than in the SCT group ( $P=0.042)$. Conclusion Considering its efficacy and safety, adjuvant SCT alone might be a reasonable choice over SCT-CRT in R0 resected pancreatic cancer.

\section{Introduction}

Pancreatic cancer occurs in 458,918 people annually worldwide [1]. The 5-year survival rate has been lower than $7 \%$ over the past 30 years; it is now $8.5 \%$, which is still low compared to other cancers [2]. Currently, pancreatic cancer is the fourth leading cause of cancer-related deaths, and it is expected to rank second by 2030 [3]. The only curative treatment is surgery; however, only $15 \%$ 20\% of patients are diagnosed in a resectable state at initial diagnosis [4]. Even after curative resection, over $80 \%$ of patients experience relapse [5].

Since curative resection is not sufficient for complete resolution of pancreatic cancer, adjuvant therapy after surgery has become a standard treatment; this has significantly increased the 5-year survival rate $[6,7]$. Based on extensive previous studies regarding diverse adjuvant treatment methods in resected pancreatic cancer [8-12], various regimens including chemoradiation therapy and chemotherapy are implemented in real clinical practice. In a clinical trial of adjuvant treatment in 2010 , there was no significant difference in efficacy between 5-fluorouracil plus folinic acid (FFA) and gemcitabine alone chemotherapy [13]. More recently, combination chemotherapy with 5-fluorouracil, leucovorin, irinotecan, and oxaliplatin (FOLFIRINOX) and gemcitabine plus capecitabine showed significantly longer survival than gemcitabine alone $[9,14]$. A definite standard chemotherapy regimen has not been established, and there are several ongoing clinical trials regarding various adjuvant chemotherapy regimens [8]. 
Currently, the National Comprehensive Cancer Network (NCCN) guidelines recommend systemic chemotherapy (SCT) or a combination of SCT and chemoradiation therapy (SCT-CRT) for resected pancreatic cancer [15]. Compared to chemotherapy, the efficacy of adjuvant chemoradiation therapy is debatable [16]. In 1985, chemoradiation therapy was first reported to increase overall survival in a clinical trial [6]. Recently, Rutter et al [14] reported that chemoradiation therapy was more effective than chemotherapy alone in resected pancreatic adenocarcinoma. Chemoradiation therapy is also known to significantly reduce loco-regional recurrence [18]. On the other hand, a recent clinical trial in Europe reported no significant difference in efficacy between chemoradiation therapy and chemotherapy groups [10]. To date, several studies regarding the efficacy of CRT have not shown consistent results, while the previous randomized controlled trials have consistently demonstrated the efficacy of adjuvant SCT [9,12$14,19]$.

Attempts to identify a standard adjuvant treatment for resected pancreatic cancer have continued, and a variety of adjuvant treatment regimens are now used in real clinical practice. However, there is still a lack of studies comparing the efficacy of SCT and SCT-CRT. We aimed to evaluate the efficacy of these treatment modalities in patients with pancreatic cancer treated with $\mathrm{R} 0$ resection.

\section{Methods}

\section{Study population}

Retrospectively, medical records of patients who underwent complete microscopic resection for pancreatic cancer at Seoul National University Hospital from September 2005 to December 2017 were reviewed. Microscopic tumor clearance (R0) was defined according to the International Union Against Cancer, and R1 was defined as the microscopic presence of tumor cells at definite resection margin [20]. After excluding patients who showed a histopathologic pattern other than pancreatic ductal adenocarcinoma, and who were treated with other active tumors within 5 years, 361 patients were analyzed. Additionally, patients who received only chemoradiation therapy, who received adjuvant therapy at other centers, and who were treated with unknown or unverified regimens of adjuvant treatment were excluded. Finally, 277 patients were included in this study (Fig. 1). All patients signed an informed consent before surgery, chemotherapy, and chemoradiation therapy. This study was approved by the Institutional Review Board of Seoul National University Hospital, Seoul, Korea (1609-015-789).

\section{Adjuvant treatment}

Patients were evaluated for residual tumor at 1 month after $\mathrm{R} 0$ resection. If there was no residual tumor, adjuvant treatment was initiated within 4 months after surgery. The adjuvant SCT and/or CRT regimen was determined through multidisciplinary discussions with each patient. Regimens for SCT group gemcitabine [12], FFA combination therapy [13], S-1 [19], and gemcitabine plus nab-paclitaxel combination therapy [NCT01964430] based on the previous clinical trials. Radiation therapy was applied 
to the tumor bed, surgical anastomosis sites, and adjacent lymph node basins at $45-55$ Gy over 5 to 8 weeks or at 20 Gy over 10 consecutive days two times repeatedly [21,22]. Chemotherapeutic agents for CRT included 5-fluorouracil and gemcitabine. The SCT-CRT group included patients who received CRT with induction and/or maintenance chemotherapy. Induction chemotherapy included gemcitabine [10] or gemcitabine and cisplatin combination therapy [NCT01396681] and maintenance chemotherapy included 5-fluorouracil [21], gemcitabine [12], and FFA combination therapy [13]. Supplementary Table S1 summarizes details of adjuvant treatment protocols according to the previous clinical trials. During the first 2 years after surgery, patients were followed up at 3 to 6 month intervals. In the absence of recurrence in the first two years, patients were evaluated every six months.

\section{Outcome measures}

The primary outcome was difference in overall survival (OS) between the SCT and SCT-CRT groups. OS was defined as time from surgery to death from any cause. Patients alive at the point of final analysis were censored at the date last seen alive. Secondary outcomes were differences in recurrence-free survival (RFS), recurrence pattern, and adverse events between the SCT and SCT-CRT groups. RFS was defined from the date of surgery to tumor recurrence, which was confirmed histologically and/or radiographically. Patients alive without tumor recurrence at the last date of follow-up or dead without evidence of tumor recurrence were censored for RFS. Loco-regional recurrence was defined as recurrence limited to the pancreas, retroperitoneum, periadventitial tissues around the resected pancreas, and regional lymph nodes. Distant recurrence was defined as recurrence beyond the loco-regional area.

During adjuvant treatment, patients were monitored closely for evidence of toxicity. Adverse events were assessed using the National Cancer Institute Common Toxicity Criteria for Adverse Events, version 4.03. Patient demographics, underlying medical conditions, and tumor marker levels were evaluated preoperatively. Pathologic findings included tumor location, diameter, differentiation, AJCC 8th edition TNM stage, distance of margin from tumor, lymphovascular invasion, and perineural invasion. Mortality data were collected from the database of Korean Ministry of the Interior and Safety.

\section{Statistical analysis}

Categorical variables were expressed as the number of patients and percentages. Continuous variables were presented as mean \pm standard deviation. Categorical data between two groups were compared using Fisher's exact test. Survival analysis was based on the Kaplan-Meier method with median and 95\% confidence interval $(\mathrm{Cl})$. Associations between several variables and OS were evaluated using Cox proportional hazard regression models calculating hazard ratios and $95 \%$ Cls. Using variables with $\mathrm{P}<0.1$ in the univariable analysis, multivariable-adjusted Cox proportional hazard regression analysis was performed. Survival differences between groups were compared using the log-rank test. Regarding the 
variation of clinicopathologic characteristics between groups, propensity score-matching analysis was performed. A caliper size of $0.2 \times \log$ (standard deviation of the propensity score) was implemented, and individual scores were calculated through logistic regression modeling based on the following 10 covariates: age, sex, ECOG, Charlson comorbidity index, safety margin, tumor histology, perineural invasion, lymphovascular invasion, AJCC stage, and CA 19-9 [22,23]. Patients receiving either SCT or SCTCRT were matched 1:1 on the propensity scores. After propensity score matching, OS, RFS, recurrence pattern, and adverse events between the two groups were examined. All statistics were evaluated using $\mathrm{R}$ version 3.5.0 for Windows (Institute for Statistics and Mathematics, Vienna, Austria; http://www.Rproject.org).

\section{Results}

\section{Patient characteristics}

Baseline characteristics are summarized in Table 1. Seventy-seven (27.8\%) patients were older than 70.

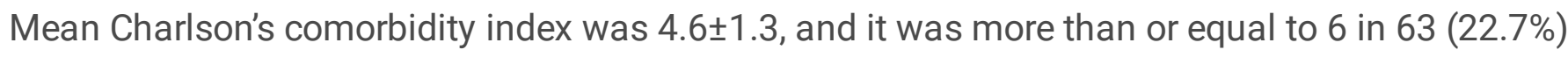
patients. AJCC stage was most commonly stage IIB in 100 (36.1\%) patients, followed by IB in 81 (29.2\%), IA in $37(13.4 \%)$, III in 53 (19.1\%), and IIA in $6(2.2 \%)$ patients. One hundred and eight (39.0\%) patients had a safety margin of less than or equal to $0.1 \mathrm{~cm}$. Preoperative CA19-9 was more than or equal to 100 $\mathrm{U} / \mathrm{mL}$ in $153(55.4 \%)$ patients. The mean follow-up duration was $32.2 \pm 28.6$ months.

\section{Prognostic factors for OS}

In total cohort, median OS was 35.1 (95\% Cl, 30.6-44.4) months. In univariable Cox proportional hazard regression analysis, variables that showed $\mathrm{P}<0.1$ included safety margin $\leq 0.1 \mathrm{~cm}$, poorly differentiated or undifferentiated histology, high AJCC stage, preoperative CA19-9 $\geq 100 \mathrm{U} / \mathrm{mL}$, and lymphovascular or perineural invasion. In multivariable-adjusted Cox proportional hazard regression analysis, lymphovascular invasion and preoperative CA19-9 level were independent prognostic factors $(P<0.001$ and $P=0.007$, respectively). The number of patients and OS classified by each variable are summarized in Table 2. In the unmatched cohort, the proportion of patients with age $\geq 70$ and safety margin $\leq 0.1 \mathrm{~cm}$ was higher in the SCT group than in the SCT-CRT. After propensity score-matching, there were no significant differences in variables associated with OS between the two groups (Table 3).

\section{Therapeutic outcomes between SCT and SCT-CRT groups in the unmatched cohort}

Adjuvant SCT was performed in 78 patients, including 44 patients receiving gemcitabine, 22 patients receiving FFA, 11 patients receiving gemcitabine plus nab-paclitaxel, and 1 patient receiving S-1. In the SCT group, the median treatment duration was $139.9 \pm 49.1$ days and 57 (73.1\%) patients completed planned adjuvant protocols. Overall, 199 patients received adjuvant SCT-CRT; the most frequent 
chemotherapeutic agent for CRT was 5-fluorouracil (119/199, 59.8\%), followed by gemcitabine (80/199, 40.2\%). In the SCT-CRT group, 51 patients underwent induction chemotherapy with gemcitabine plus cisplatin and 10 patients with gemcitabine. After CRT, patients received gemcitabine most frequently (116/199, 58.3\%), followed by FFA $(70 / 199,35.2 \%)$ for maintenance adjuvant chemotherapy. The median treatment duration was $196.9 \pm 46.7$ days in the SCT-CRT group, and 153 (76.9\%) patients completed planned adjuvant protocols. The median treatment duration was significantly longer in the SCT-CRT group than in the SCT group $(P<0.001)$, while there was no significant difference in completion rate of planned regimens between the two groups $(P=0.534)$.

Median OS in the SCT group and the SCT-CRT group was 31.1 (95\% Cl, 26.9-47.4) months and 35.9 (95\% $\mathrm{Cl}, 30.7-47.8)$ months, respectively $(\mathrm{P}=0.681)$. During follow-up, recurrence occurred in $170(61.4 \%)$ patients, and median RFS was 16.8 (95\% $\mathrm{Cl} 15.0-21.9)$ months. There was no significant difference in RFS between the SCT and SCT-CRT groups $(15.4$ [95\% Cl, 11.6-24.8] months and $17.7[95 \% \mathrm{Cl}, 15.0-23.6]$ months, respectively; $P=0.453)$. Figure $2 A$ and $2 B$ illustrate the differences in $O S$ and $R F S$ between the SCT and SCT-CRT groups in the unmatched cohort.

\section{Therapeutic outcomes between SCT and SCT-CRT groups in the propensity score-matched cohort}

The propensity score-matched cohort was comprised of 142 patients; 71 patients each were classified as SCT group and SCT-CRT group. The median OS was 35.1 (95\% Cl, 26.9-82.1) months in the SCT group and $34.8(95 \% \mathrm{Cl}, 26.2-69.2)$ months in the SCT-CRT group ( $\mathrm{P}=0.993)$. The median RFS of the patients who underwent SCT was $15.4(95 \% \mathrm{Cl}, 12.1-31.1)$ months, which was not significant different from that of patients who received SCT-CRT (17.0 [95\% $\mathrm{Cl}, 13.2-33.9]$ months; $\mathrm{P}=0.789)$. Figure $3 \mathrm{~A}$ and $3 \mathrm{~B}$ show the differences in OS and RFS between the two groups in the propensity score-matched cohort

Differences in the recurrence pattern between SCT and SCT-CRT groups are summarized in Table 4. Recurrence was confirmed in 40 (56.3\%) patients in the SCT group and $43(60.6 \%)$ in the SCT-CRT group $(P=0.734)$. There were no significant differences in the incidence of loco-regional and distant recurrence between the two groups $(P=0.833$ and $P=0.491$, respectively). The liver was the most common site of distant recurrence in both groups. There was no significant difference in the number of patients who could undergo further lines of treatment after relapse between SCT and SCT-CRT groups $(42.1 \%$ and $47.9 \%$, respectively; $\mathrm{P}=0.613$ ).

Details of adverse events greater than or equal to moderate grade during adjuvant treatment are summarized in Table 5. No somatic adverse events were significantly different between the two groups. The incidences of neutropenia, anemia, and hypertransaminesemia were higher in the SCT-CRT group than in the SCT group. In total, adverse events greater than or equal to moderate grade occurred more frequently in the SCT-CRT group than in the SCT group $(P=0.042$.

\section{Discussion}


A wide variety of regimens has been studied as adjuvant treatment for resected pancreatic cancer [8-10]. However, adjuvant treatment for pancreatic cancer is not yet standardized. There are discrepancies among previous studies comparing the efficacy of adjuvant CRT and SCT $[6,10,17]$. This study demonstrated no significant differences in OS and RFS between the SCT and the SCT-CRT groups. In addition, there were no significant differences in recurrence pattern between the two groups. Adverse events greater than or equal to moderate grade occurred more frequently in the SCT-CRT group than in the SCT group.

CRT did not show a significant increase in OS compared with the control group in ESPAC-1 and EORTC clinical trials $[10,24,25]$. On the other hand, recent studies showed that CRT combined with SCT was superior to SCT alone $[17,26]$. However, recent large-scale studies are limited by lacking of pathologic information including surgical free margin, T stage, and N stage $[17,26]$. In this study, SCT-CRT did not show significantly better survival outcomes compared with SCT alone in both the unmatched and the propensity score-matched cohorts. These results are consistent with the previous clinical trials that did not demonstrate the significant efficacy of CRT $[10,24,25]$.

CRT is known to lower the risk of loco-regional relapse, whereas SCT reduces distant metastasis $[27,28]$. A previous study reported $\sim 20 \%$ local only recurrence in resected pancreatic cancer [29]. In this study, local only recurrence was more frequent in the SCT group, but the difference was not statistically significant. This is consistent with a previous clinical trial, indicating that conventional RT dose may not be sufficient for improving loco-regional relapse [25]. There are many types of radiation therapies currently available for pancreatic cancer, including new modalities such as stereotactic body radiation therapy $[30,31]$. Efforts should be made to find the most effective radiotherapy method to reduce local recurrence when combined with SCT.

A previous study showed that there is no significant difference in efficacy between gemcitabine and FFA combination chemotherapy [13]. In JASPAC 01 trial, S-1 showed significantly higher 5-years overall survival rate than gemcitabine in Asian population [19]. Most recently, FOLFIRINOX showed a significant increase in OS compared with gemcitabine single therapy, and adverse events greater than or equal to severe grade occurred in $~ 3 / 4$ of patients [14]. Nab-paclitaxel, which has recently shown significant therapeutic effects in metastatic pancreatic cancer, is being studied to confirm its effectiveness in resected pancreatic cancer [NCT01964430]. Based on these clinical trials, this study included patients receiving gemcitabine, FFA, gemcitabine plus nab-paclitaxel, or S-1 in the chemotherapy group. Since various adjuvant treatment regimens have different spectra of efficacy and toxicity, appropriate patient stratification is essential to establish a proper treatment plan.

Surgical margins, lymph node metastasis, tumor differentiation, high level of preoperative CA19-9, and perineural and lymphovascular invasion are well-known prognostic factors in resected pancreatic cancer [32]. In accordance with previous studies, this study demonstrated several independent prognostic factors for OS based on the Cox proportional hazard regression model. Patients should be thoroughly evaluated 
on the basis of an elaborate prognostication model to establish active treatment plans, such as intensive systemic chemotherapy or clinical trials, in high-risk patients.

The limitations of this study are as follows. First, there is an inherent selection bias due to its singlecenter retrospective study design. Second, the statistical power may be less than ideal because there was a relatively small total number of patients in this study. Third, this study involved heterogeneous chemotherapeutic regimens, and since our institution is active in radiation therapy, the proportion of patients who received SCT was relatively small. However, adjuvant treatment protocols that were verified in previous clinical trials were included in this study. Additionally, propensity score-matched analysis was performed between the two groups to reduce the bias from confounding variables. Since this study is limited by the small sample size and retrospective design, a well-designed clinical trial that prospectively compares SCT and/or CRT should be followed.

In conclusion, in R0 resected pancreatic cancer, there were no significant differences in OS, RFS, and recurrence pattern between SCT and SCT-CRT groups while SCT-CRT group showed significantly longer treatment duration and higher incidence of adverse events than SCT group. In terms of efficacy and safety, SCT alone might be a reasonable choice over SCT-CRT in R0 resected pancreatic cancer.

\section{Abbreviations}

PDAC, pancreatic ductal adenocarcinoma; FFA, 5-fluorouracil and folinic acid; FOLFIRINOX, 5-fluorouracil, leucovorin, irinotecan, and oxaliplatin; AJCC, American Joint Committee on Cancer; CRT, chemoradiation therapy; SCT, systemic chemotherapy; OS, overall survival; RFS, recurrence-free survival; $\mathrm{Cl}$, confidence interval

\section{Declarations}

\section{Author's contributions}

Conception and design by Ji Kon Ryu; provision of study materials or patients by Young Hoon Choi and Bang-sup Shin; organizing and conversion of data by Min su You, Young Hoon Choi and Bang-sup Shin; data analysis and interpretation by Woo Hyun Paik, Sang Hyub Lee and Yong-Tae Kim; manuscript writing by Min su You and Ji Kon Ryu. All authors read and approved the final manuscript.

\section{Ethics approval and consent to participate}

This study was approved by Institutional Review Board of the Seoul National University Hospital, Seoul, Korea (1609-015-789). Patients were not required to give informed consent to the study because the analysis used anonymous clinical data that were obtained after each patient agreed to treatment by written consent.

\section{Availability of data and materials}


The datasets used and/or analysed during the current study are available from the corresponding author on reasonable request.

\section{Consent for publication}

Not applicable.

\section{References}

1. Bray F, Ferlay J, Soerjomataram I, Siegel RL, Torre LA, Jemal A. Global cancer statistics 2018 : GLOBOCAN estimates of incidence and mortality worldwide for 36 cancers in 185 countries. CA Cancer J Clin. 2018;68(6):394-424.

2. Surveillance, Epidemiology, and End Results Program. SEER Stat Facts Sheets: Pancreas Cancer, Bethesda, MD, USA. 2018.

3. Rahib L, Smith BD, Aizenberg R, Rosenzweig AB, Fleshman JM, Matrisian LM. Projecting cancer incidence and deaths to 2030: the unexpected burden of thyroid, liver, and pancreas cancers in the United States. Cancer Res. 2014;74(11):2913-21.

4. Gillen S, Schuster T, Meyer Zum Buschenfelde C, Friess H, Kleeff J. Preoperative/neoadjuvant therapy in pancreatic cancer: a systematic review and meta-analysis of response and resection percentages. PLoS Med. 2010;7(4):e1000267.

5. Kleeff J, Korc M, Apte M, La Vecchia C, Johnson CD, Biankin AV, et al. Pancreatic cancer. Nat Rev Dis Primers. 2016;2:16022.

6. Kalser MH, Ellenberg SS. Pancreatic cancer. Adjuvant combined radiation and chemotherapy following curative resection. Archives of surgery. 1985;120(8):899-903.

7. Roberts KJ. Improving outcomes in patients with resectable pancreatic cancer. BJS. 2017;104(11):1421-3.

8. Neoptolemos JP, Kleeff J, Michl P, Costello E, Greenhalf W, Palmer DH. Therapeutic developments in pancreatic cancer: Current and future perspectives. Nat Rev Gastroenterol Hepatol. 2018;15(6):33348.

9. Neoptolemos JP, Palmer DH, Ghaneh P, Psarelli EE, Valle JW, Halloran CM, et al. Comparison of adjuvant gemcitabine and capecitabine with gemcitabine monotherapy in patients with resected pancreatic cancer (ESPAC-4): a multicentre, open-label, randomised, phase 3 trial. Lancet. 2017;389(10073):1011-24.

10. Laethem J-LV, Hammel P, Mornex F, Azria D, Tienhoven GV, Vergauwe P, et al. Adjuvant Gemcitabine Alone Versus Gemcitabine-Based Chemoradiotherapy After Curative Resection for Pancreatic Cancer: A Randomized EORTC-40013-22012/FFCD-9203/GERCOR Phase II Study. J Clin Oncol. 2010;28(29):4450-6.

11. Ueno H, Kosuge T, Matsuyama Y, Yamamoto J, Nakao A, Egawa S, et al. A randomised phase III trial comparing gemcitabine with surgery-only in patients with resected pancreatic cancer: Japanese 
Study Group of Adjuvant Therapy for Pancreatic Cancer. Br J Cancer. 2009;101(6):908-915.

12. Oettle H, Post S, Neuhaus P, Gellert K, Langrehr J, Ridwelski K, et al. Adjuvant chemotherapy with gemcitabine vs observation in patients undergoing curative-intent resection of pancreatic cancer: a randomized controlled trial. JAMA. 2007;297(3):267-277.

13. Neoptolemos JP, Stocken DD, Bassi C, Ghaneh P, Cunningham D, Goldstein D, et al. Adjuvant chemotherapy with fluorouracil plus folinic acid vs gemcitabine following pancreatic cancer resection: A randomized controlled trial. JAMA. 2010;304(10):1073-81.

14. Conroy T, Hammel P, Hebbar M, Ben Abdelghani M, Wei AC, Raoul JL, et al. FOLFIRINOX or Gemcitabine as Adjuvant Therapy for Pancreatic Cancer. N Engl J Med. 2018;379(25):2395-406

15. National Comprehensive Cancer Network. Pancreatic adenocarcinoma (Version 1.2019).

16. Twombly R. Adjuvant chemoradiation for pancreatic cancer: few good data, much debate. J Natl Cancer Inst. 2008;100(23):1670-1.

17. Rutter CE, Park HS, Corso CD, Lester-Coll NH, Mancini BR, Yeboa DN, Johung KL. Addition of radiotherapy to adjuvant chemotherapy is associated with improved overall survival in resected pancreatic adenocarcinoma: An analysis of the National Cancer Data Base. Cancer. 2015;121(23):4141-9.

18. Regine WF, Winter KA, Abrams R, Safran H, Hoffman JP, Konski A, et al. Fluorouracil-based chemoradiation with either gemcitabine or fluorouracil chemotherapy after resection of pancreatic adenocarcinoma: 5-year analysis of the U.S. Intergroup/RTOG 9704 phase III trial. Ann Surg Oncol. 2011;18(5):1319-26.

19. Uesaka K, Boku N, Fukutomi A, Okamura Y, Konishi M, Matsumoto I, et al. Adjuvant chemotherapy of S-1 versus gemcitabine for resected pancreatic cancer: a phase 3 , open-label, randomised, noninferiority trial (JASPAC 01). Lancet. 2016;388(10041):248-257.

20. Schlitter AM, Esposito I. Definition of microscopic tumor clearance (r0) in pancreatic cancer resections. Cancers. 2010;2(4):2001-2010.

21. Neoptolemos JP, Dunn JA, Stocken DD, Almond J, Link K, Beger H, et al. Adjuvant chemoradiotherapy and chemotherapy in resectable pancreatic cancer: a randomised controlled trial. Lancet. 2001;358(9293):1576-85.

22. Austin PC. The performance of different propensity score methods for estimating marginal hazard ratios. Stat Med. 2013;32(16):2837-2849.

23. Austin PC. Optimal caliper widths for propensity-score matching when estimating differences in means and differences in proportions in observational studies. Pharm Stat. 2011;10(2):150-161.

24. Neoptolemos JP, Stocken DD, Friess H, Bassi C, Dunn JA, Hickey H, et al. A randomized trial of chemoradiotherapy and chemotherapy after resection of pancreatic cancer. $\mathrm{N}$ Engl J Med. 2004;350(12):1200-10.

25. Klinkenbijl JH, Jeekel J, Sahmoud T, van Pel R, Couvreur ML, Veenhof $\mathrm{CH}$, et al. Adjuvant Radiotherapy and 5-Fluorouracil After Curative Resection of Cancer of the Pancreas and 
Periampullary Region: Phase III Trial of the EORTC Gastrointestinal Tract Cancer Cooperative Group. Ann Surg. 1999;230(6):776.

26. Hsieh MC, Chang WW, Yu HH, Lu CY, Chang CL, Chow JM, et al. Adjuvant radiotherapy and chemotherapy improve survival in patients with pancreatic adenocarcinoma receiving surgery: adjuvant chemotherapy alone is insufficient in the era of intensity modulation radiation therapy. Cancer Med. 2018;7(6):2328-38.

27. Ryan DP, Hong TS, Bardeesy N. Pancreatic Adenocarcinoma. N Engl J Med. 2014;371(11):1039-49.

28. Wang F, Kumar P. The role of radiotherapy in management of pancreatic cancer. J Gastrointest Oncol. 2011;2(3):157-67.

29. Griffin JF, Smalley SR, Jewell W, Paradelo JC, Reymond RD, Hassanein RE, Evans RG. Patterns of failure after curative resection of pancreatic carcinoma. Cancer. 1990;66(1):56-61.

30. Goldsmith C, Plowman PN, Green MM, Dale RG, Price PM. Stereotactic ablative radiotherapy (SABR) as primary, adjuvant, consolidation and re-treatment option in pancreatic cancer: scope for dose escalation and lessons for toxicity. Radiat Oncol. 2018;13(1):204.

31. Zhong J, Patel K, Switchenko J, Cassidy RJ, Hall WA, Gillespie T, et al. Outcomes for patients with locally advanced pancreatic adenocarcinoma treated with stereotactic body radiation therapy versus conventionally fractionated radiation. Cancer. 2017;123(18):3486-93.

32. Bilici A. Prognostic factors related with survival in patients with pancreatic adenocarcinoma. World $\mathrm{J}$ Gastroenterol. 2014;20(31):10802-12.

\section{Figures}




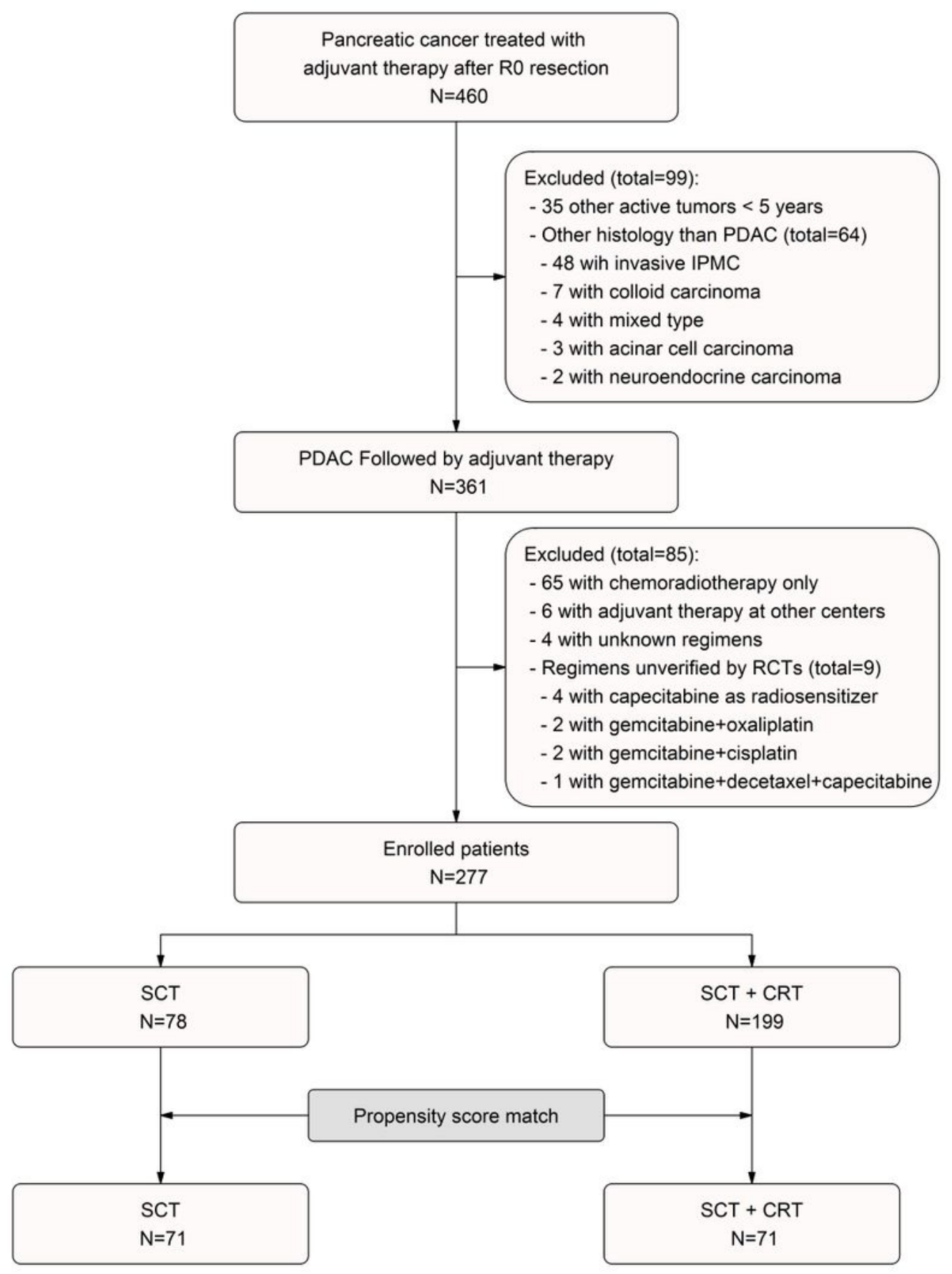

\section{Figure 1}

Flowchart of patient inclusion. PDAC, pancreatic ductal adenocarcinoma; IPMC, intraductal papillary mucinous neoplasm; RCT, randomized controlled trial; SCT, systemic chemotherapy; SCT-CRT, combination therapy with chemoradiation and systemic chemotherapy 

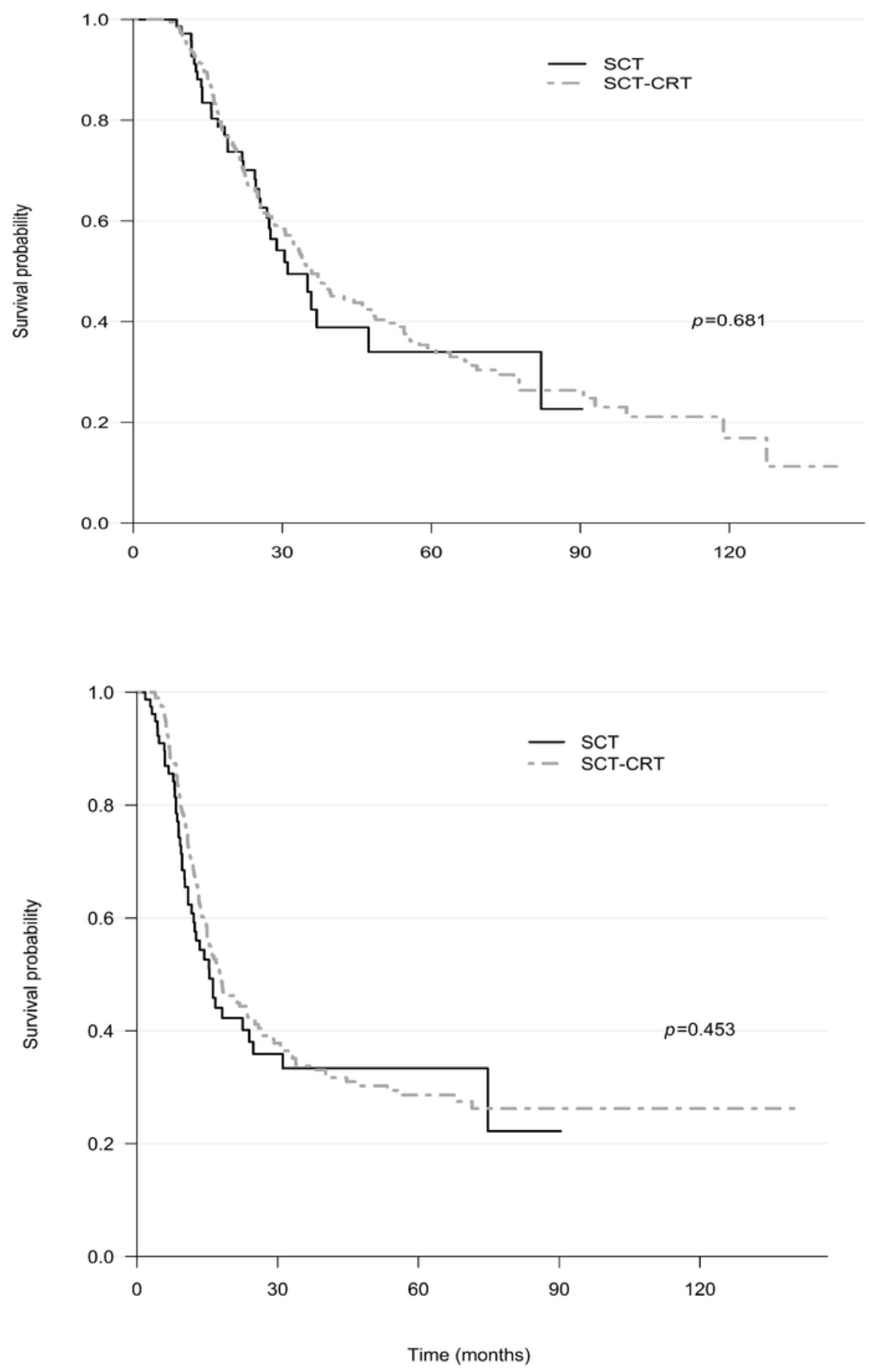

Figure 2

Kaplan-Meier estimates of (A) overall survival and (B) recurrence-free survival, classified by SCT and SCT-CRT in the unmatched cohort 

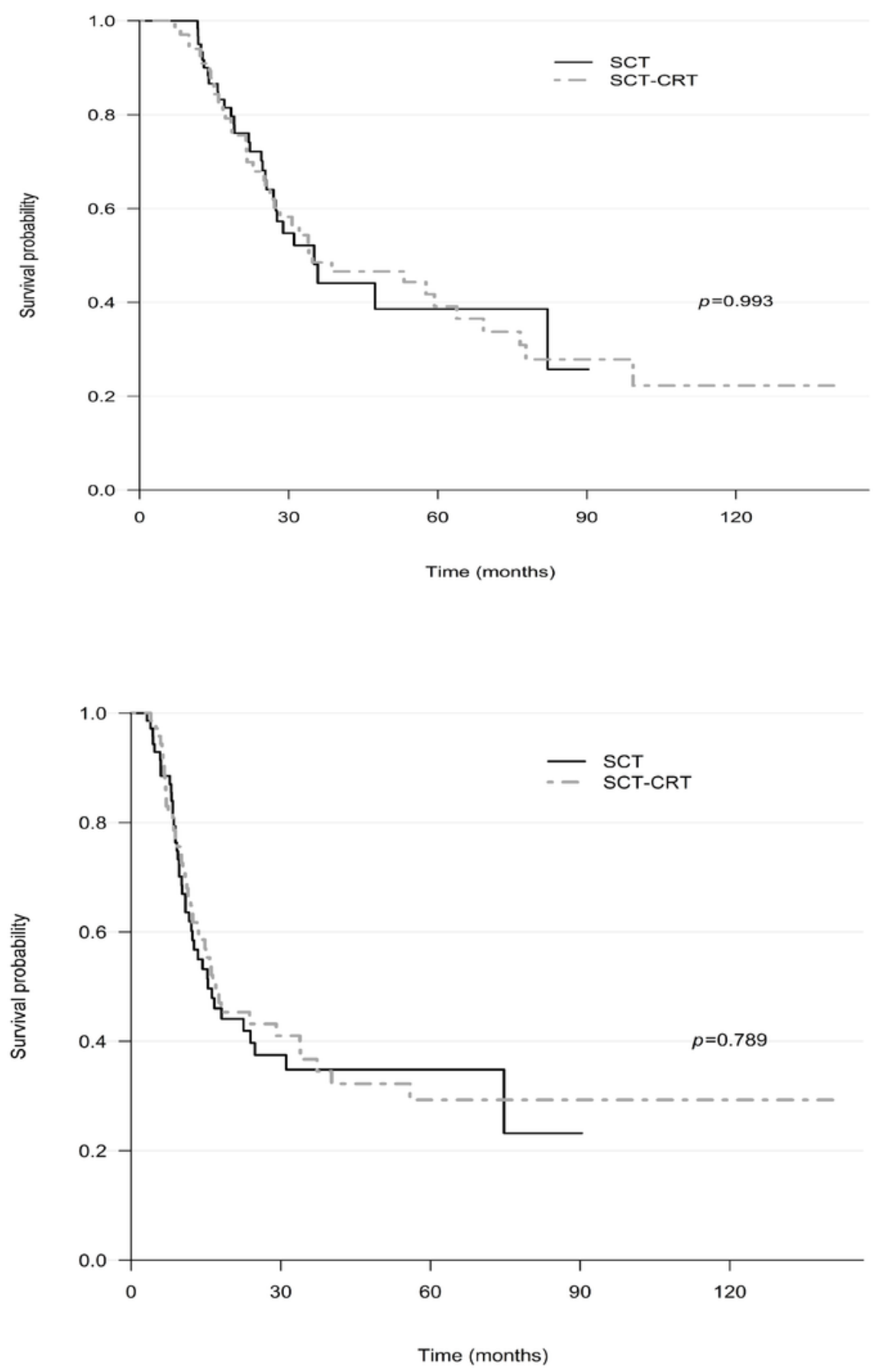

Figure 3

Kaplan-Meier estimates of (A) overall survival and (B) recurrence-free survival, classified by SCT and SCT-CRT in the propensity score-matched cohort

\section{Supplementary Files}


This is a list of supplementary files associated with this preprint. Click to download.

- SupplementaryTables.pdf

- Tables.pdf 